\title{
Effective treatment of polydatin weakens the symptoms of collagen-induced arthritis in mice through its anti-oxidative and anti-inflammatory effects and the activation of MMP-9
}

\author{
BO LI and XIAO-LI WANG
}

Pediatric Surgery of Second People's Hospital of Liaocheng City, Linqing, Shandong 252600, P.R. China

Received September 30, 2015; Accepted April 18, 2016

DOI: $10.3892 / \mathrm{mmr} .2016 .5903$

\begin{abstract}
Polydatin is a natural extract used in traditional Chinese medicine, which leads to a marked improvement in the microcirculation perfusion and enhances the animal myocardial contraction force. The present study aimed to determine whether an effective treatment of polydatin ameliorates the symptoms of collagen-induced arthritis (CIA), and also to explore the potential mechanism. Male DBA/1J mice were induced into CIA model mice. The administration of polydatin effectively suppressed CIA in mice. The serum levels of malondialdehyde (MDA), superoxide dismutase (SOD), tumor necrosis factor- $\alpha$ (TNF- $\alpha$ ) and interleukin $1 \beta$ (IL-1 $\beta$ ) were effectively increased following the induction of CIA in the model mice compared with the control group. The elevated serum levels of MDA, SOD, TNF- $\alpha$ and IL- $1 \beta$ were markedly suppressed by the effective treatment of polydatin in CIA mice, compared with the CIA model group. However, an increase in the level of matrix metalloproteinase-9 (MMP-9) was markedly induced in the CIA mice compared with the control group. As compared with the CIA group, the expression of MMP-9 was substantially reduced by the effective treatment of polydatin. Taken together, the effective treatment of polydatin ameliorated the symptoms of CIA through an exertion of its antioxidative and anti-inflammatory effects, and also via activation of the expression of matrix metalloproteinase-9 (MMP-9) in mice.
\end{abstract}

\section{Introduction}

Rheumatoid arthritis (RA) is a chronic progressive autoimmune disease characterized by multi-joint synovial inflammation and the presence of a large number of T lymphocytes within the synovial tissue infiltrate. CD4 ${ }^{+}$

Correspondence to: Mr. Bo Li, Pediatric Surgery of Second People's Hospital of Liaocheng City, 306 Jiankang Street, Linqing, Shandong 252600, P.R. China

E-mail: bolince@163.com

Key words: polydatin, collagen-induced arthritis, anti-oxidative, anti-inflammatory, MMP-9
T lymphocytes exert the predominant pathogenic role in the development of RA (1). Previous studies have identified that, in the limb joints of a mouse RA model, the mice have the same T-cell receptor (TCR) V $\beta$ clone type T cells. However, as the development of the disease progresses, the numbers of this type of clone $\mathrm{T}$ cell increase gradually, whereas those of other TCR V $\beta$ clone type T cells are gradually reduced: Therefore, this $\mathrm{T}$ cell clone type may be associated with the pathological changes of arthritis (1-3). Collagen-induced arthritis (CIA) is associated with pathogenetic and pathological changes that are similar to those of RA, and so CIA is often used as a model of human RA (4).

The chemical name of polydatin is 3,4,5-trihydroxy stilbene-3- $\beta$-D-glycosidase; since there are three hydroxyl phenolic groups in the structure of polydatin, the compound readily reacts with oxidizing material and functions as an effective antioxidant, scavenging the effects of free radicals (5). Polydatin is also a monomer, exerting antiviral and antibacterial effects, and it is a natural extract used in traditional Chinese medicine [it has been extracted from Japanese knotweed, Reynoutria Japonica (Houtt), in our School]. Post-lab studies of giant knotweed have determined that this plant possesses evident curative properties for the treatment of blood loss, burns and septic shock, and it may improve the survival rate of animals in a state of shock by improving the microcirculation perfusion and enhancing the animals' myocardial contractile force $(5,6)$.

The present study aimed to determine whether an effective treatment of polydatin ameliorates the symptoms of CIA, and also to explore the potential mechanisms involved. The results have revealed, to our knowledge for the first time, that the effective treatment of polydatin ameliorates the symptoms of CIA through an exertion of its antioxidative and anti-inflammatory effects, and also via activation of the expression of matrix metalloproteinase-9 (MMP-9), in mice.

\section{Materials and methods}

Materials. Polydatin (purity $\geq 95 \%$, as determined by high-pressure liquid chromatography; structure shown in Fig. 1), bovine collagen type II (CII), complete Freund's adjuvant (CFA), incomplete Freund's adjuvant (IFA), malondialdehyde (MDA), glutathione (GSH), tumor necrosis factor- $\alpha$ (TNF- $\alpha$ ), interleukin-6 (IL-6), specific enzyme-linked 
immunosorbent assay kits and the caspase-3/9 fluorometric assay kit were acquired from Sigma-Aldrich (St. Louis, MO, USA).

Animals and induction of CIA. Male DBA/1J mice (age, 6-7 weeks) were purchased from the laboratory of Shandong University (Shandong, China), housed in a controlled environment $\left(22 \pm 2^{\circ} \mathrm{C}, 12 \mathrm{~h} \mathrm{light/dark} \mathrm{cycle)} \mathrm{and} \mathrm{provided} \mathrm{with}\right.$ standard rodent chow and tap-water. Experiments involving the mice were performed in accordance with the Guide for the Care and Use of Laboratory Animals, adopted by The Second People's Hospital of Liaocheng City (Liaocheng, Shandong, China). Bovine CII ( $2 \mathrm{mg} / \mathrm{ml})$, an equal volume of CFA and $2 \mathrm{mg} / \mathrm{ml}$ Mycobacterium tuberculosis H37Ra were mixed together. The mice were intradermally injected with $100 \mu \mathrm{l}$ of the emulsion containing $100 \mu \mathrm{g}$ CII, and subsequently were administered booster injections with $100 \mu \mathrm{g}$ CII in IFA after 21 days of primary immunization.

Division into groups. Animals were randomly divided into five groups: i) Control group, in which the mice were intradermally injected with $0.5 \mathrm{ml}$ normal saline; ii) CIA group, in which CIA mice were intradermally injected with $0.5 \mathrm{ml}$ normal saline; iii) 'polydatin 15' group, in which CIA mice were intradermally injected with $15 \mathrm{mg} / \mathrm{kg}$ polydatin for $24 \mathrm{~h}$; iv) 'polydatin 30' group, in which CIA mice were intradermally injected with $30 \mathrm{mg} / \mathrm{kg}$ polydatin for $24 \mathrm{~h}$; v) and 'polydatin 45' group, in which CIA mice were intradermally injected with $45 \mathrm{mg} / \mathrm{kg}$ polydatin for $24 \mathrm{~h}$.

Evaluating CIA progression. Paw swelling was evaluated by measuring the thickness of two hind paws using a digital caliper. The severity of the arthritis was evaluated using a clinical scoring system on a scale of 0-4 for each paw: 0 , no signs of arthritis; 1 , swelling and/or redness in one joint; 2 , swelling and/or redness in more than one joint; 3 , swelling and/or redness in the entire paw; and 4, severe swelling of the entire paw with deformity and/or ankylosis. The total arthritis score produced a maximum score of 16 , as the sum of each score of the four limbs.

Evaluating oxidative damage and reactive inflammation. The blood samples were collected from an intracardiac puncture and subsequently were centrifuged for $20 \mathrm{~min}$ at $2,000 \mathrm{x} \mathrm{g}$ at $4^{\circ} \mathrm{C}$ (Heraeus Pico 17/21; Thermo Fisher Scientific, Inc., Waltham, MA, USA). The levels of MDA, GSH, TNF- $\alpha$ and IL-6 were analyzed using specific enzyme-linked immunosorbent assay kits in accordance with the manufacturer's protocol (Sigma-Aldrich).

Western blot analysis for MMP-9 and B-cell lymphoma 2 (Bcl-2)/ Bcl-2-associated X protein (Bax). The arthritic tissue samples were collected and homogenized with $100 \mu \mathrm{l}$ tissue lysis buffer (Beyotime Institute of Biotechnology, Nanjing, China) for $30 \mathrm{~min}$ on ice. Protein concentrations were determined using the bicinchoninic acid (BCA) method (Nanjing KeyGen Biotech Co., Ltd., Nanjing, China). An equal quantity of protein was dissolved in a $10 \%$ sodium dodecyl sulfate polyacrylamide gel for electrophoresis, and subsequently transferred onto fluoride membranes $(0.22 \mathrm{~mm}$;

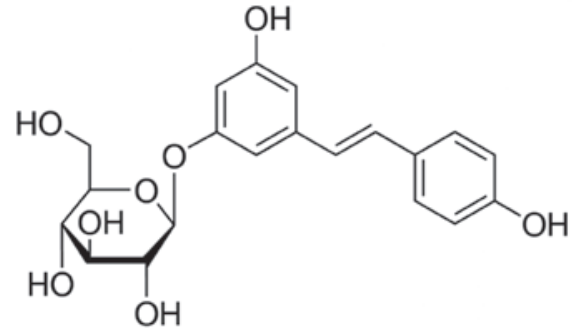

Figure 1. The chemical structure of polydatin.

Bio-Rad Laboratories, Inc., Hercules, CA, USA). The fluoride membranes were blocked with $5 \%$ skim milk powder in Tris-buffered saline-Tween 20 for $1 \mathrm{~h}$ at room temperature and incubated with mouse monoclonal anti-MMP-9 $(1: 1,000$; cat. no. sc-13520), mouse monoclonal anti-Bcl-2 (1:1,000; cat. no. sc-7382), mouse monoclonal anti-Bax (1:1,000; cat. no. sc-23959) and mouse monoclonal anti- $\beta$-actin $(1: 1,000$; cat. no. sc-47778) antibodies (1:1,000; all purchased from Santa Cruz Biotechnology, Inc. (Dallas, TX, USA) overnight at $4^{\circ} \mathrm{C}$. Subsequently, the membranes were incubated with horseradish peroxidase (HRP)-conjugated goat anti-mouse secondary antibody (1:5,000; Beyotime Institute of Biotechnology; cat. no. A0216) and the bands were visualized using an enhanced chemiluminescence kit (Tianjin Sungene Biotech Co., Ltd.). Relative protein expression was determined using Quantity One software (version 4.62; Bio-Rad Laboratories, Inc.).

Evaluating caspase-3/9 activity. The arthritic tissue samples were collected and homogenized in $100 \mu 1$ tissue lysis buffer (Beyotime Institute of Biotechnology) for $30 \mathrm{~min}$ on ice. Protein concentrations were determined using the BCA method (Nanjing KeyGen Biotech Co., Ltd.). An equal quantity of protein was evaluated using caspase-3/9 fluorometric assay kits, in accordance with the manufacturer's protocol (Sigma-Aldrich).

Statistical analysis. Data are expressed as the mean \pm standard deviation, and compared by one-way analysis of variance using the least significant difference multiple-comparison test. SPSS 19.0 statistical software (IBM SPSS, Armonk, NY, USA) was used for the data analysis. $\mathrm{P}<0.05$ was considered to indicate a statistically significant difference.

\section{Results}

Effective treatment of polydatin on CIA in mice. To evaluate the effects of polydatin on CIA in mice, the clinical arthritis score and hind-paw thickness were evaluated to determine the effectiveness of the treatment on CIA. These indexes were markedly higher compared with those of the control group, although the increases were reduced on treatment with polydatin in a dose-dependent manner. The difference was revealed to be statistically significant $(\mathrm{P}<0.01)$ following the treatment with 30 or $45 \mathrm{mg} / \mathrm{kg}$ polydatin for the measurement of the clinical arthritis score, and for the $45 \mathrm{mg} / \mathrm{kg}$ polydatin treatment alone in the case of the hind-paw thickness (Fig. 2). 
Effective treatment of polydatin against oxidative damage in CIA mice. Subsequently, the effectiveness of polydatin as an antioxidant, working against oxidative damage in CIA mice, was examined. The levels of MDA and GSH were analyzed to evaluate the effectiveness of the treatment on CIA. In the CIA group mice, the MDA level was markedly increased, and that of GSH was markedly decreased, compared with the control group (Fig. 3). However, the levels of MDA and GSH in CIA mice were markedly suppressed and promoted by pretreatment with 30 or $45 \mathrm{mg} / \mathrm{kg}$ polydatin compared with the model group (Fig. 3).

Effective treatment of polydatin on reactive inflammation in CIA mice. The effectiveness of polydatin in preventing reactive inflammation in CIA mice was subsequently investigated. The serum levels of TNF- $\alpha$ and IL- $1 \beta$ were analyzed to evaluate the effectiveness of polydatin treatment on CIA. A marked elevation in the serum levels of TNF- $\alpha$ and IL- $1 \beta$ were observed in the CIA group mice compared with the control group (Fig. 4). As expected, the elevation in the serum levels of TNF- $\alpha$ and IL-1 $\beta$ were significantly reduced on treatment with polydatin (30 or $45 \mathrm{mg} / \mathrm{kg}$; $\mathrm{P}<0.01$ ) compared with the model group (Fig. 4).

Effective treatment of polydatin on MMP-9 in CIA mice. Subsequently, the effect of treatment of polydatin on MMP-9 was investigated in CIA mice. As shown in Fig. 5, the induction of CIA markedly inhibited the protein expression of MMP-9 in CIA model mice compared with the control group. However, the addition of 30 or $45 \mathrm{mg} / \mathrm{kg}$ polydatin led to a marked increase in the protein expression of MMP-9 in CIA mice compared with the model group (Fig. 5).

Effective treatment of polydatin on caspase-3/9 in CIA mice. The effectiveness of the treatment of polydatin against apoptosis in CIA mice was subsequently analyzed by measuring the activity of caspases-3 and 9. The induction of CIA markedly induced the activities of caspases-3 and 9 in the CIA model mice compared with the control group (Fig. 6). Furthermore, the CIA-induced activities of caspases-3 and 9 were markedly suppressed on treatment with 30 or $45 \mathrm{mg} / \mathrm{kg}$ polydatin compared with the model group (Fig. 6).

Effective treatment of polydatin on Bcl-2/Bax in CIA mice. It is well known that the relative levels of $\mathrm{Bcl}-2 / \mathrm{Bax}$ provide an important indicator of apoptosis. In this experiment, the protein expression of Bcl-2 and Bax was analyzed by western blot analysis. Compared with the control group, the protein expression of Bcl-2 was markedly reduced, and that of the Bax was markedly increased, in CIA mice (Fig. 7). However, treatment with 30 or $45 \mathrm{mg} / \mathrm{kg}$ polydatin significantly ameliorated the changes in the CIA model mice compared with the model group $(\mathrm{P}<0.01 ;$ Fig. 7$)$.

\section{Discussion}

RA is a chronic systemic autoimmune disease, and its pathological basis is joint synovitis (4). RA, of unknown etiology and with complex and varied clinical manifestations, can cause serious damage to human health (4). Type II collagen is a protein that is responsible for organization, predominantly existing in the articular cartilage and eye tissue $(3,7)$. It is the protein that is featured predominantly in the composition of the articular cartilage. Damage to the joints results in the release of type II collagen, which stimulates an autoimmune response, and this is considered to be one of the mechanisms underpinning the development of RA $(2,7)$. Through the release of type II collagen, mice are able to induce an autoimmune response, leading to the erosion of cartilage in type II collagen-mediated multi-arthritis, which is known as CIA (8). The characteristics of CIA are similar in several ways to those of human RA, including synovitis, the formation of cartilage and subchondral pannus, inflammatory cell infiltration, the erosion of cartilage, bone resorption and remodeling, and so forth (9). Therefore, CIA is regarded as a unique animal model of human RA, which is therefore widely used in studies on the mechanism of RA. In the present study, treatment with 30 or $45 \mathrm{mg} / \mathrm{kg}$ polydatin markedly inhibited the clinical arthritis score and hind-paw thickness in CIA mice.

Free radicals are involved in the occurrence and development of RA, in the process of synovial inflammatory lesions of the joints, and in bone destruction; additionally, free radicals are directly or indirectly involved in synovial membrane and bone injuries $(8,9)$. All types of free radicals are involved in this process: Hydroxyl free radicals biodegrade proteoglycan, hypochlorous acid leads to the fracture of the collagen, and the capability of hydrogen peroxide to diffuse throughout the tissue leads to the inhibition of cartilage glycoprotein synthesis and obstruction of the synthesis of ATP. Hydrogen peroxide also exerts an effect on the glycolytic enzyme, glyceraldehyde-3-phosphate dehydrogenase, in the cartilage cells, accelerating proteolytic hydrolysis and mediating the degradation of the cartilage by free radicals (10). The reactivity of hypochlorous acid, $\mathrm{O}_{2}$ - and vitamin $\mathrm{C}$ has a great influence on the function of cartilage, resulting in a reduction in the vitamin $\mathrm{C}$ content in joint synovial fluid. Activated phagocytes produce reactive oxygen species, which are able to alter the immunofluorescent properties of immunoglobulin $\mathrm{G}(\mathrm{IgG})$, leading to the further activation of phagocyte fluorescent protein polymers $(11,12)$. The degeneration of $\mathrm{IgG}$ inhibits rheumatoid factor, directly resulting in the generation of C-reactive protein (12). This type of reaction manifests itself in the rheumatoid joints in the long term, demonstrating that free radicals exert an important role in the process of chronic inflammation (13). In the present study, the effective treatment of 30 or $45 \mathrm{mg} / \mathrm{kg}$ polydatin markedly reduced oxidative damage in CIA mice. Ji et al (14) demonstrated that treatment with polydatin ameliorates blood-brain barrier permeability through suppression of oxidative stress in the permanent middle cerebral artery occlusion rat brain (14). In agreement with previous studies, a study by Miao et al (15) demonstrated that polydatin protects against ischemia/reperfusion injury through antioxidative stress mechanisms (15). The findings in the present study have also revealed that the effectiveness of polydatin as an antioxidative agent has an important role in RA treatment.

$\mathrm{RA}$ is a type of chronic, systemic autoimmune disease which has the predominant feature of symmetrical polyarthritis, and anti-inflammatory analgesic symptomatic 

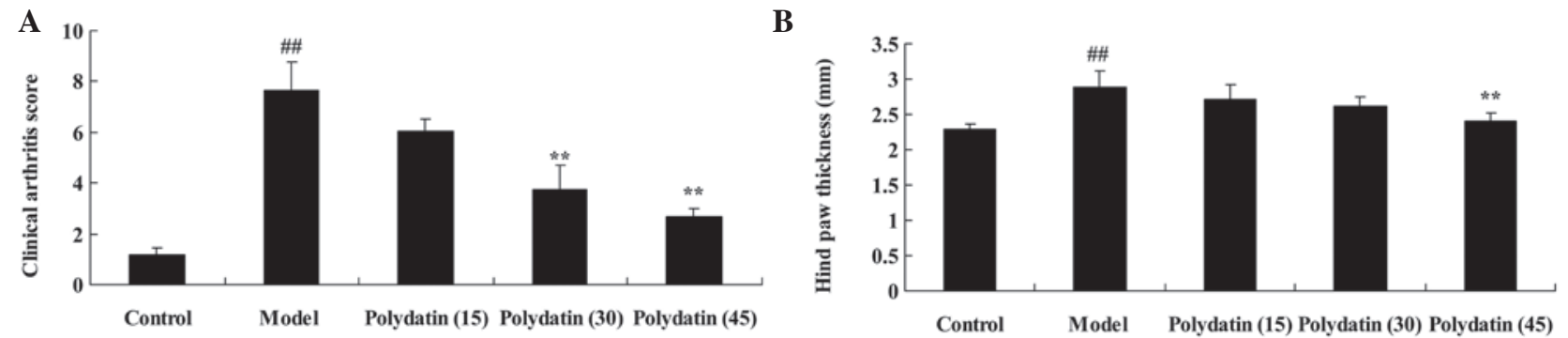

Figure 2. Effective treatment of polydatin on collagen-induced arthritis in mice. Effective treatment of polydatin on (A) clinical arthritis score and (B) hind-paw thickness in collagen-induced arthritis mice. ${ }^{\# \#} \mathrm{P}<0.01$ compared with the control group; ${ }^{* *} \mathrm{P}<0.01$ compared with the model group.
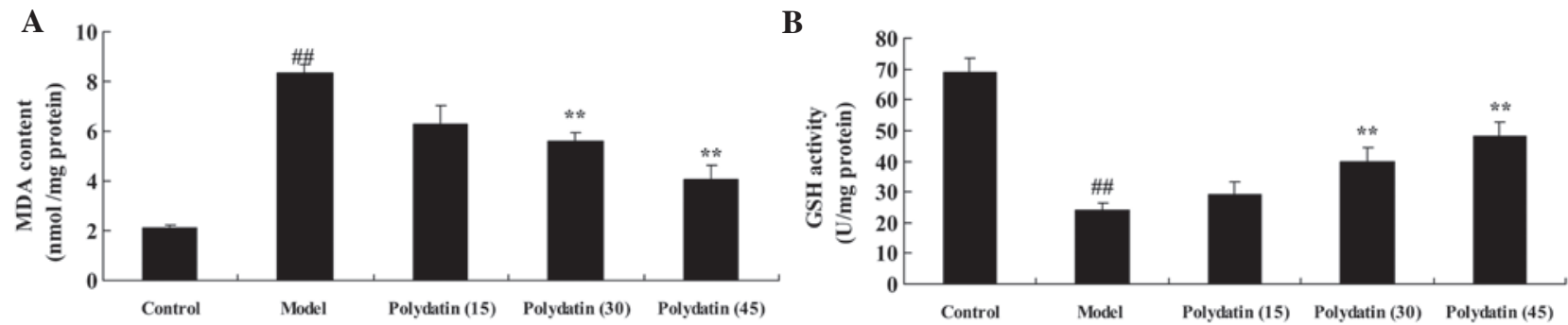

Figure 3. Effective treatment of polydatin on oxidative damage in collagen-induced arthritic mice. Effective treatment of polydatin on the levels of (A) MDA and (B) GSH in collagen-induced arthritic mice is shown. ${ }^{\# \#} \mathrm{P}<0.01$ compared with the control group; ${ }^{* *} \mathrm{P}<0.01$ compared with the model group. GSH, glutathione; MDA, malondialdehyde.
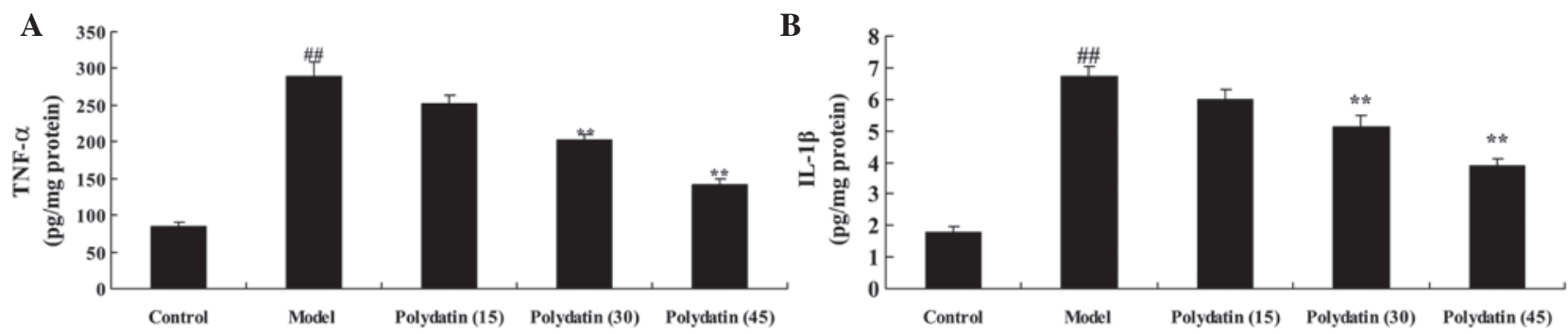

Figure 4. Effective treatment of polydatin on reactive inflammation in collagen-induced arthritic mice. Effective treatment of polydatin on the serum levels of (A) TNF- $\alpha$ and (B) IL-6 in collagen-induced arthritic mice is shown. ${ }^{\# \#} \mathrm{P}<0.01$ compared with the control group; ${ }^{* *} \mathrm{P}<0.01$ compared with the model group. TNF- $\alpha$, tumor necrosis factor- $\alpha$; and IL-6, interleukin-6.

treatments, which would delay the development of the disease, offer the major therapeutic options $(2,16)$. Local inflammation of the joints is a key link in the process of its pathogenesis; bone loss or damage caused by inflammation is the result of changes to the joints in RA, and symptoms of pain associated with joint swelling are often the most severe symptoms of which the patient complains (9). Therefore, inhibiting the inflammation has become one of the predominant targets of the clinical treatment (16). In the present study, the administration of polydatin ( 30 or $45 \mathrm{mg} / \mathrm{kg}$ ) markedly ameliorated reactive inflammation in CIA mice. In a previous study, Chen et al (17) elucidated that the effective treatment of polydatin elicited prominent nephroprotective activities via oxidative stress and inflammatory responses in fructose-induced urate nephropathic mice. Lou et al (18) provided evidence that polydatin inhibits nitric oxide and prostaglandin $\mathrm{E}_{2}$ production in lipopolysaccharide-stimulated RAW 264.7 cells, and also exerts potent anti-inflammatory activity in macrophages. These results suggested that the effective treatment of polydatin against RA is associated with its anti-inflammatory response in CIA mice.

MMPs are responsible for the degradation of one or several types of extracellular matrix, belonging to the zinc-dependent peptidase enzyme family (19). Previous studies have shown that the degradation of the extracellular matrix that is associated predominantly with collagenous connective tissue may promote pannus hyperplasia, leading to the destruction of the cartilage, ligaments and bone, and the gelatinases, MMP-2 and MMP-9, are very closely associated with the collagenous connective tissue reaction $(19,20)$. MMP-9 acts on elastin and type II and type V collagens, further degrading the collagen enzyme degradation products. MMP-9 has been demonstrated to dissolve the cartilage collagen (21). MMP-9 is the most widely expressed of all the MMPs, and it is of great importance to the physiological metabolism of connective tissue (22). MMP-9 is not only capable of dissolving collagen types I and II, but it can also degrade gelatin, an excessive production of which eventually 
A



B

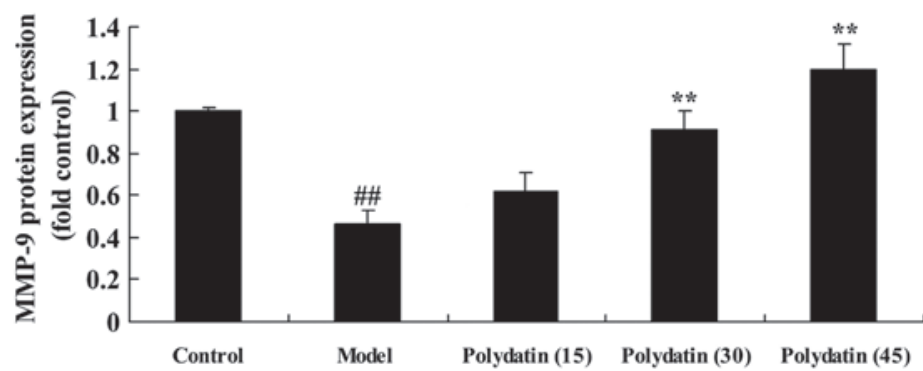

Figure 5. Effective treatment of polydatin on MMP-9 in collagen-induced arthritic mice. Effective treatment of polydatin on the protein expression of MMP-9 was investigated by (A) western blot analysis, and (B) the statistical analysis of MMP-9 protein expression in collagen-induced arthritic mice is shown. ${ }^{\# \prime} \mathrm{P}<0.01$ compared with the control group; ${ }^{* *} \mathrm{P}<0.01$ compared with the model group. MMP- 9 , matrix metalloproteinase 9.
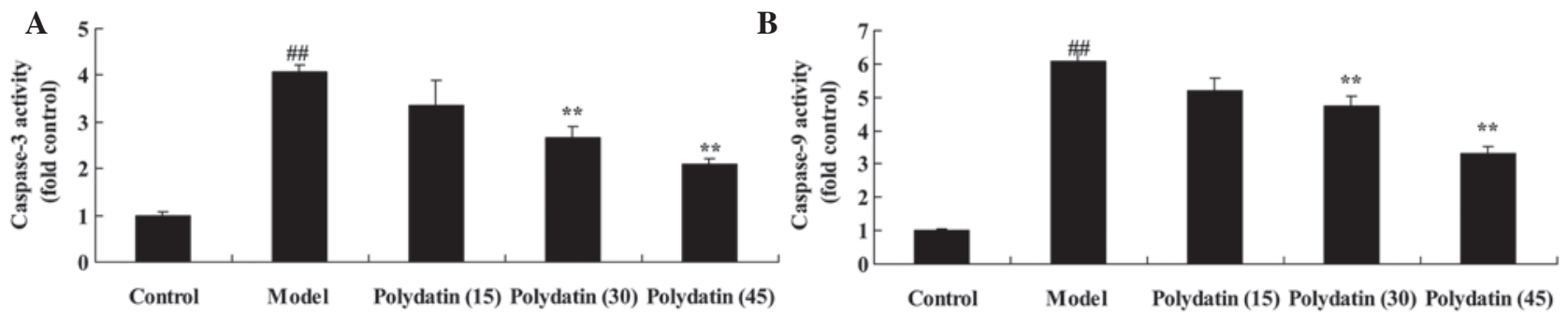

Figure 6. Effective treatment of polydatin on caspases-3/9 in collagen-induced arthritic mice. Effective treatment of polydatin on the activities of (A) caspase-3 and (B) caspase-9 in collagen-induced arthritic mice is shown. ${ }^{\# \#} \mathrm{P}<0.01$ compared with the control group; ${ }^{* *} \mathrm{P}<0.01$ compared with the model group.

A

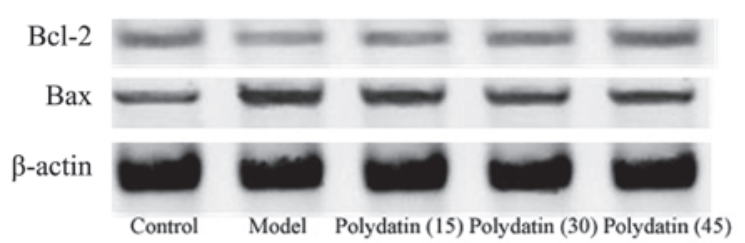

B

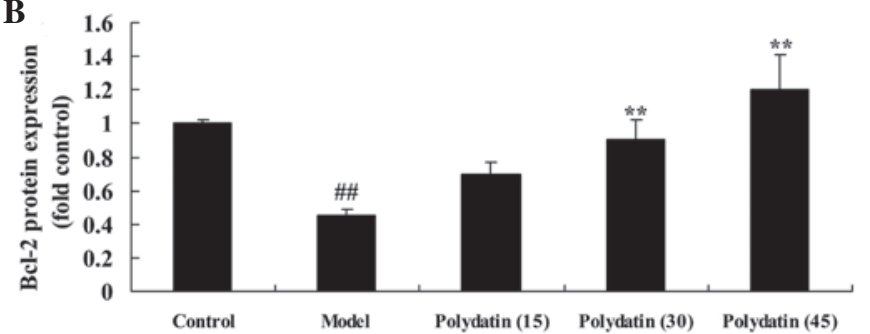

C

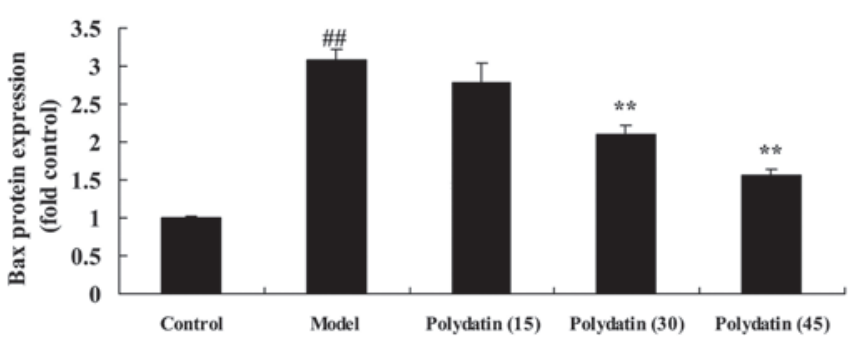

Figure 7. Effective treatment of polydatin on Bcl-2/Bax in collagen-induced arthritic mice. Effective treatment of polydatin on the (A) protein expression of Bcl-2/Bax was determined by western blot analysis, and the statistical analysis of the protein expression of (B) Bcl-2 and (C) Bax in collagen-induced arthritic mice is shown. ${ }^{\#} \mathrm{P}<0.01$ compared with the control group; ${ }^{* *} \mathrm{P}<0.01$ compared with the model group. Bcl-2, B-cell lymphoma 2 ; Bax, Bcl-2-associated $\mathrm{X}$ protein.

leads to arthritis (23). Animal experiments revealed that an inhibitor of the MMPs was able to prevent destruction of the joints (22). The results in the present study have shown that treatment with 30 or $45 \mathrm{mg} / \mathrm{kg}$ polydatin markedly increased the protein expression of MMP-9, increased the activities of caspases-3 and 9, and upregulated the Bcl-2/Bax signaling pathway in CIA mice. In addition, Zhang et al (24) reported that polydatin was able to reduce the expression of MMP-9 in the aortas of apolipoprotein E double-knockout mice. Li et al (25) revealed that polydatin ameliorates burn-induced lung injury through caspase-3 activity and Bax. The findings of the present study demonstrated that treatment of the mice with polydatin against CIA exerted an antiapoptotic effect and was associated with an increase in the expression of MMP-9, although the precise mechanism has yet to be fully elucidated.

In conclusion, the effective treatment of polydatin alleviated symptoms of the disease of CIA, as demonstrated by its antioxidative and anti-inflammatory properties, the activation of MMP-9 and suppression of caspases-3/9, and 
upregulation of the $\mathrm{Bcl}-2 / \mathrm{Bax}$ pathway. In particular, the present study has identified a suitable target for the further scientific investigation of RA.

\section{References}

1. Ohnishi Y, Tsutsumi A, Matsumoto I, Goto D, Ito S, Kuwana M, Uemura Y, Nishimura Y and Sumida T: Altered peptide ligands control type II collagen-reactive $\mathrm{T}$ cells from rheumatoid arthritis patients. Mod Rheumatol 16: 226-228, 2006.

2. Chang JM, Cheng CM, Hung LM, Chung YS and Wu RY: Potential use of plectranthus amboinicus in the treatment of rheumatoid arthritis. Evid Based Complement Alternat Med 7: 115-120, 2010.

3. Furuzawa-Carballeda J, Macip-Rodríguez P, Galindo-Feria AS, Cruz-Robles D, Soto-Abraham V, Escobar-Hernández S, Aguilar D, Alpizar-Rodríguez D, Férez-Blando K and Llorente L: Polymerized-type I collagen induces upregulation of Foxp3-expressing CD4 regulatory T cells and downregulation of IL-17-producing CD4 ${ }^{+} \mathrm{T}$ cells (Th17) cells in collagen-induced arthritis. Clin Dev Immunol 2012: 618608, 2012.

4. Wang H, Chen W, Wang L, Li F, Zhang C and Xu L: Tumor necrosis factor receptor-associated factor 6 promotes migration of rheumatoid arthritis fibroblast-like synoviocytes. Mol Med Rep 11: 2761-2766, 2015.

5. Du QH, Peng $\mathrm{C}$ and Zhang H: Polydatin: A review of pharmacology and pharmacokinetics. Pharm Biol 51: 1347-1354, 2013.

6. Liu LT, Guo G, Wu M and Zhang WG: The progress of the research on cardio-vascular effects and acting mechanism of polydatin. Chin J Integr Med 18: 714-719, 2012.

7. Rosloniec EF, Ivey RA III, Whittington KB, Kang AH and Park HW: Crystallographic structure of a rheumatoid arthritis MHC susceptibility allele, HLA-DR1 (DRB1*0101), complexed with the immunodominant determinant of human type II collagen. J Immunol 177: 3884-3892, 2006.

8. Wu D, Chen J, Zhu H, Xiong XG, Liang QH, Zhang Y, Zhang Y, Wang Y, Yang B and Huang X: UPLC-PDA determination of paeoniflorin in rat plasma following the oral administration of Radix Paeoniae Alba and its effects on rats with collagen-induced arthritis. Exp Ther Med 7: 209-217, 2014.

9. Li QH, Xie WX, Li XP, Huang KT, Du ZH, Cong WJ, Zhou LH, Ye TS and Chen JF: Adenosine A2A receptors mediate anti-inflammatory effects of electroacupuncture on synovitis in mice with collagen-induced arthritis. Evid Based Complement Alternat Med 2015: 809560, 2015.

10. Park KH, Mun CH, Kang MI, Lee SW, Lee SK and Park YB: Treatment of collagen-induced arthritis using immune modulatory properties of human mesenchymal stem cells. Cell Transplant, 2015 (Epub ahead of print).

11. Suh SJ, Kim KS, Kim MJ, Chang YC, Lee SD, Kim MS, Kwon DY and Kim CH: Effects of bee venom on protease activities and free radical damages in synovial fluid from type II collagen-induced rheumatoid arthritis rats. Toxicol In Vitro 20: 1465-1471, 2006.

12. Jones NR, Pegues MA, McCrory MA, Singleton W, Bethune C, Baker BF, Norris DA, Crooke RM, Graham MJ and Szalai AJ: A Selective inhibitor of human C-reactive protein translation is efficacious in vitro and in C-reactive protein transgenic mice and humans. Mol Ther Nucleic Acids 1: e52, 2012.

13. Wang Y, Sun W, Chen L, Xu X, Wu Y, Zhang J and Zhang Y: Anti-arthritic activity of Fu-Fang-Lu-Jiao-Shuang on collagen-induced arthritis in Balb/c mice and its underlying mechanisms. Pharmacogn Mag 11: 242-249, 2015.
14. Ji H, Zhang X, Du Y, Liu H, Li S and Li L: Polydatin modulates inflammation by decreasing NF- $\kappa$ B activation and oxidative stress by increasing Gli1, Ptch1, SOD1 expression and ameliorates blood-brain barrier permeability for its neuroprotective effect in pMCAO rat brain. Brain Res Bull 87: 50-59, 2012.

15. Miao Q, Wang S, Miao S, Wang J, Xie Y and Yang Q: Cardioprotective effect of polydatin against ischemia/reperfusion injury: Roles of protein kinase $\mathrm{C}$ and mito K(ATP) activation. Phytomedicine 19: 8-12, 2011.

16. Ferrandiz ML, Maicas N, Garcia-Arnandis I, Terencio MC, Motterlini R, Devesa I, Joosten LA, van den Berg WB and Alcaraz MJ: Treatment with a $\mathrm{CO}$-releasing molecule (CORM-3) reduces joint inflammation and erosion in murine collagen-induced arthritis. Ann Rheum Dis 67: 1211-1217, 2008.

17. Chen L, Lan Z, Lin Q, Mi X, He Y, Wei L, Lin Y, Zhang Y and Deng $X$ : Polydatin ameliorates renal injury by attenuating oxidative stress-related inflammatory responses in fructose-induced urate nephropathic mice. Food Chem Toxicol 52: 28-35, 2013.

18. Lou T, Jiang W, Xu D, Chen T and Fu Y: Inhibitory effects of polydatin on lipopolysaccharide-stimulated RAW 264.7 cells. Inflammation 38: 1213-1220, 2015

19. Lou G, Gao Y, Ning XM and Zhang QF: Expression and correlation of CD44v6, vascular endothelial growth factor, matrix metalloproteinase-2 and matrix metalloproteinase-9 in Krukenberg tumor. World J Gastroenterol 11: 5032-5036, 2005.

20. Catanzaro R, Marotta F, Jain S, Rastmanesh R, Allegri F, Celep G, Lorenzetti A, Polimeni A and Yadav H: Beneficial effect of a sturgeon-based bioactive compound on gene expression of tumor necrosis factor- $\alpha$, matrix metalloproteinases and type-10 collagen in human chondrocytes. J Biol Regul Homeost Agents 26: 337-345, 2012.

21. Vitlianova K, Georgieva J, Milanova M and Tzonev S: Blood pressure control predicts plasma matrix metalloproteinase-9 in diabetes mellitus type II. Arch Med Sci 11: 85-91, 2015

22. Babichenko II, Andriukhin MI, Pulbere S and Loktev A: Immunohistochemical expression of matrix metalloproteinase- 9 and inhibitor of matrix metalloproteinase- 1 in prostate adenocarcinoma. Int J Clin Exp Pathol 7: 9090-9098, 2014.

23. Surlin P, Oprea B, Solomon SM, Popa SG, Moța M, Mateescu GO, Rauten AM, Popescu DM, Dragomir LP, Puiu I, et al: Matrix metalloproteinase $-7,-8,-9$ and -13 in gingival tissue of patients with type 1 diabetes and periodontitis. Rom J Morphol Embryol 55 (Suppl 3): S1137-S1141, 2014.

24. Zhang JC, Chen KJ and Zheng GJ: Regulatory effect of Chinese herbal compound for detoxifying and activating blood circulation on expression of NF- $\mathrm{KB}$ and MMP-9 in aorta of apolipoprotein E gene knocked-out mice. Zhongguo Zhong Xi Yi Jie He Za Zhi 27: 40-44, 2007 (In Chinese).

25. Li T, Cai S, Zeng Z, Zhang J, Gao Y, Wang X and Chen Z: Protective effect of polydatin against burn-induced lung injury in rats. Respir Care 59: 1412-1421, 2014. 